\title{
Importancia de la laparoscopia en el diagnóstico de la enfermedad pélvica inflamatoria
}

\author{
Hernando Gaitán Duarte*; Edith Angel Müller**; Ariel Ruiz Parra***
}

\begin{abstract}
RESUMEN: Con el objeto de investigar la sensibilidad de los hallazgos clínicos en el diagnóstico de la EPI, se analizaron 76 pacientes que ingresaron al Servicio de Sépticas del IMI de Bogotá durante el período comprendido entre el $1^{\circ}$ de noviembre de 1990 y el 31 de diciembre de 1991, quienes fueron sometidas a un procedimiento quirúrgico (laparoscopia [48 pacientes], laparotomía [17 pacientes] y ambos procedimientos [11 pacientes]) para establecer el diagnóstico de certeza de la enfermedad.
\end{abstract}

En 66 de estas 76 pacientes $(\mathbf{8 6 . 8 \%})$ se estableció el diagnóstico de EPI. Los diagnósticos de las otras 10 pacientes fueron: embarazo ectópico en tres; apendicitis en tres; pelvis normal en dos y quiste del ligamento ancho y perforación uterina con pelviperitonitis en una paciente cada uno.

En el $86 \%$ de los casos con sospecha clínica de EPI, ésta fue confirmada. Se encontró un $11 \%$ de falsos positivos con otra patología y un $2.8 \%$ con pelvis normal. En este grupo de pacientes analizando el diagnóstico clínico de ingreso y el diagnóstico de certeza (establecido por laparoscopia y/o laparotomía), encontramos que los hallazgos clínicos tuvieron una sensibilidad del $92 \%$ y un valor predictivo positivo del $85 \%$.

El enfoque quirúrgico fue conservador: en las pacientes con EPI grado I, no se realizó ninguna laparotomía; de las pacientes con EPI grados II y III sólo requirieron salpingooforectomía unilateral 16 ( $24.2 \%$ del total de pacientes con EPI) y en las demás pacientes únicamente se realizó lavado peritoneal.

PALABRAS CLA VES: Laparoscopia, enfermedad pélvica inflamatoria.

SUMMARY: To stablish the sensitivity of the clinical diagnosis of pelvic inflammatory disease (PID), we analyzed 76 patients, of the Gynecology and Obstetrics Infectious Disease Service in the "Instituto Materno Infantil" of Bogotá, during the period between november 1st, 1990 to december 31, 1991. In these patients the clinical diagnosis was verified during a surgical procedure: laparoscopy in 48; laparotomy in 17 and both procedures in 11 patients.

The diagnosis of PID, was confirmed in $66(86.8 \%)$ of the 76 patients who were hospitalized with a clinical diagnosis of PID. The findings in the other 10 women were: ectopic pregnancy in three, appendicitis in three, normal pelvis in two, and parovarian cyst and uterine perforation with pelvic peritonitis in one patient each.

We found no differences in age, occupation, number of gestations, parity and number of abortions. The number of sexual partners was 2.1 in the PID group and 1.2 in the non-PID group. We found that the actual or previous use of intrauterine device was higher in the group of patients with PID $(62.6 \%$ vs. $28.6 \%$; $=0.04) .14 .8 \%$ of the patients with PID, have had an anterior episode of the disease, while in the other group this antecedent was negative.

The classic symptoms and signs described in women with salpingitis were found in both groups in similar percentages; nevertheless we can't calculate the specificity because of the absence of a control group.

In $86 \%$ of the cases with clinical suspicion of PID, the diagnosis was confirmed. We found a false positive diagnosis in $13.8 \%$, $(11 \%$ patients with other pathologies and $2.8 \%$ with normal pelvis). In these patients the clinical diagnosis of PID had a sensitivity of.92\% and a predictive positive value of $85 \%$.

Laparoscopy is the most reliable and sensitive method for the diagnosis of PID. However it is expensive, not always is available and because of the important number of patients with PID in our Institution it isn't practical on clinical grounds. For these reasons the diagnostic basis remain the clinical criteria in our service.

KEY WORDS: Laparoscopy, pelvic inflamatory disease.

El término de enfermedad pélvica inflamatoria (EPI) se aplica en forma general a la afección infecciosa de las trompas de falopio, ovarios y/o de las estructuras adyacen-

\footnotetext{
* Profesor Asistente de Ginecología y Obstetricia U. Nal. de Col. Coordinador del Servicio de Sépticas Instituto Materno Infantil. ** Instructora Asociada de Ginecología y Obstetricia. U. Nal. de Col. *** . Profesor Asistente de Ginecología y Obstetricia. U. Nal. de Col.
}

tes. Las complicaciones y secuelas de la enfermedad son múltiples y graves e incluyen: formación de abscesos, evolución hacia la enfermedad crónica, adherencias pélvicas, infertilidad por factor tubárico y síndromes de dolor pélvico crónico, entre otras. La importancia del estudio de la EPI radica no sólo en sus secuelas, sino también en su alta incidencia; se considera que en los Estados Unidos alcanzó proporciones "casi epidémicas" en la década de los 80 (1). 
En el Servicio de Sépticas del Instituto Materno Infantil de Bogotá (IMI), el 14\% de las pacientes fueron hospitalizadas con el diagnóstico de EPI en el año de 1989. El 75\% de estas pacientes tenían menos de 30 años y el $65 \%$ eran nuligestantes o tenían un solo hijo.

En el IMI se han realizado previamente estudios sobre la enfermedad pélvica inflamatoria (2-4) tradicionalmente se ha iniciado el tratamiento de la EPI una vez que se ha establecido la impresión diagnóstica con base en los hallazgos de la historia clínica y de la exploración física (5-7). Se conoce por los datos de la literatura que el examen clínico solamente tiene una sensibilidad entre el 65 y el $70 \%$ para el diagnóstico de la salpingitis cuando se compara con la laparoscopia. Jacobson y cols. (8) encontraron en 1969 que un número importante de salpingitis cursa con muy pocos síntomas y, algunas pacientes con el cuadro clínico típico no tenían evidencia de enfermedad al examen laparoscópico; recomendando el uso generalizado de la laparoscopia ya que un diagnóstico más temprano y preciso mejora el pronóstico de la enfermedad (8). Desde entonces la laparoscopia es considerada como el método de elección para el diagnóstico de la enfermedad. El examen permite, no sólo la visualización de la pelvis, sino también la toma de muestras para estudio bacteriológico (9-10).

Los criterios clínicos que se han establecido para el diagnóstico de EPI son: (11): 3 criterios mayores, que siempre deben estar presentes: dolor hipogástrico a la palpación, dolor a la movilización del cérvix y dolor a la palpación de los anexos. Y criterios menores, de los cuales uno o más deben estar presentes: temperatura de $38^{\circ} \mathrm{C} \mathrm{o}$ más, material purulento obtenido por culdocentesis o laparoscopia, absceso pélvico al examen físico o por ultrasonido, leucocitosis mayor de $10.500 / \mathrm{mm}^{3}$, VSG $>15$ $\mathrm{mm} /$ hora, evidencia de colonización cervical por gonococo o clamidia y presencia de más de 5 leucocitos/campo de 100X en el Gram de secreción endocervical.

En este estudio se usó la siguiente clasificación clínica de la EPI (12):

Grado I. No complicada (limitada a trompas y ovarios).

Grado II. Complicada (masa o absceso involucrando trompas y/u ovarios). Con o sin peritonitis pélvica.

Grado III. Diseminada a estructuras extrapélvicas (absceso tubo-ovárico roto) o con respuesta sistémica.

De acuerdo con los hallazgos laparoscópicos o quirúrgicos se usó la siguiente clasificación de la enfermedad (8):

Leve: Trompas libres con eritema y edema, sin exudado purulento espontáneo, pero que puede aparecer al manipular las trompas.

Moderada: Presencia de material purulento, eritema y edema más marcados. Las trompas pueden estar fijas y el orificio de la fimbria puede estar obstruido.

Severa: Piosálpinx o absceso.

\section{Materiales y métodos}

El diseño de este estudio fue observacional, descriptivo y prospectivo (respecto a la direccionalidad).

La investigación se desarrolló en el Servicio de Sépticas del Instituto Materno Infantil de Bogotá. Este hospital es una institución de tercer nivel adscrita a la Facultad de Medicina de la Universidad Nacional de Colombia, que atiende pacientes de bajos recursos económicos.
En este estudio se analizaron 76 pacientes quienes ingresaron al Servicio de Sépticas con impresión diagnóstica de EPI (con base en los criterios mencionados) o a quienes se les estableció dicho diagnóstico definitivo de egreso, durante el período comprendido entre el $1^{\circ}$ de noviembre de 1990 y el 31 de diciembre de 1991. Estas pacientes fueron sometidas a procedimientos quirúrgicos (laparotomía y/o laparoscopia) que permitieron establecer el diagnóstico de certeza, ya que estos se consideran como el "estándar de oro" para el diagnóstico de EPI.

A todas las pacientes se les realizó una historia clínica y un examen físico completos y se investigaron particularmente los factores de riesgo. Todos los datos del ingreso y de la evolución posterior se consignaron en un formulario previamente diseñado.

Los exámenes de laboratorio que se realizaron a estas pacientes fueron: cuadro hemático completo con VSG, parcial de orina, serología, química sanguínea (creatinina, nitrógeno ureico, glucosa, proteínas séricas totales y diferenciales) y frotis de secreción cervical. En algunas pacientes se realizaron cultivos de muestras obtenidas por culdocentesis, laparoscopia o laparotomía. Se practicó estudio de ultrasonido pélvico en $66 \%$ de pacientes.

Los datos se presentan como promedios $\pm \mathrm{DE}$ y proporciones. Se aplicó la prueba exacta de Fisher y la prueba de $\mathrm{X}^{2}$ cuando fue apropiado para las comparaciones estadísticas. Todo el procesamiento de los datos se realizó mediante el programa EPI-INFO 5.01 - STAT-CALC.

Este estudio se diseñó con el fin de determinar la situación actual de la salpingitis aguda en la población que asiste al IMI de Bogotá. Describir los núcleos de población más afectados, los factores de riesgo de nuestras pacientes e investigar el valor de los datos clínicos y de laboratorio en el diagnóstico de la EPI en comparación con los hallazgos laparoscópicos y/o de laparotomía.

\section{Resultados}

En 66 de estas 76 pacientes $(86.8 \%)$ se confirmó el diagnóstico de EPI. Los diagnósticos que se establecieron en las otras 10 pacientes fueron: embarazo ectópico en tres; apendicitis en tres; pelvis normal en dos y quiste del ligamento ancho y perforación uterina con pelviperitonitis en una paciente cada uno.

Las características generales de las pacientes, como edad, número de partos y número de abortos, son similares en ambos grupos, según se puede observar en la tabla 1.

\section{Tabla 1}

CARACTERISTICAS GENERALES DE LAS PACIENTES CON DIAGNOSTICO VISUAL (IMI 1990-1991)

\begin{tabular}{|l|r|r|}
\hline & CON EPI & SIN EPI \\
\hline Edad (años) & $30.2 \pm 8.7$ & $30.5 \pm 8.7 *$ \\
Paridad & $2.6 \pm 1.7$ & $2.2 \pm 1.8 *$ \\
Abortos & $0.4 \pm 0.8$ & $0.5 \pm 0.7 *$ \\
\hline \\
Promedio \pm DE \\
NSS
\end{tabular}

En cuanto a la ocupación, en el grupo de pacientes con EPI el $68 \%$ estaban dedicadas al hogar, el $28.4 \%$ eran 
empleadas y $3.6 \%$ (2 pacientes) eran prostitutas. En las pacientes sin EPI el $50 \%$ estaban dedicadas al hogar y el $50 \%$ eran empleadas.

Con respecto a los factores considerados de riesgo para la enfermedad encontramos que el promedio de compañeros sexuales fue de 2.1 para el grupo de pacientes con EPI y de 1.2 para las pacientes sin EPI. La fracción de dispariclad (Odds ratio, OR) para un compañero sexual fue de 1 y para dos compañeros sexuales fue de 1.12 ( $p=0.34$; NS). Un $66.7 \%$ y un $87.5 \%$ de las pacientes con EPI y sin EPI. respectivamente, utilizaba algún método de anticoncepción. Encontramos un uso significativamente mayor del DIU (actual o previo) en el grupo de EPI que en las pacientes sin EPI (62.6\% vs. $28.6 \%$; $=0.04)$. Un $14.8 \%$ de las pacientes del grupo de EPI habían tenido un episodio anterior de la enfermedad, mientras que este antecedente fue negativo en el grupo de pacientes sin EPI. El antecedente de leucorrea fue referido por un $67.8 \%$ de las pacientes con EPI y un $57.1 \%$ de las pacientes sin EPI. En las pacientes con EPI la serología fue reactiva en el $13.8 \%$ y, en los frotis endocervicales se encontraron diplococos gram negativos intra y extracelulares en un $6.9 \%$ de los casos; mientras que, en el grupo de pacientes sin EPI estos hallazgos fueron negativos (Tabla 2 ).

Tabla 2

FACTORES DE RIESGO DE LAS PACIENTES CON DIAGNOSTICO VISUAL (IMI 1990-1991)

\begin{tabular}{|c|c|c|}
\hline Factores de riesgo & EPI & Sin EPI \\
\hline Leucorrea & $67.8 \%$ & $57.1 \% *$ \\
\hline Antecedente de EPI & $14.8 \%$ & $0.0 \% *$ \\
\hline Anticoncepción & $66.7 \%$ & $87.5 \% *$ \\
\hline Anovulatorios & $8.1 \%$ & $0.0 \% *$ \\
\hline DIU & $62.6 \%$ & $28.6 \% * *$ \\
\hline Otros & $29.3 \%$ & $71.4 \% *$ \\
\hline Serología reactiva & $13.8 \%$ & $0.0 \% *$ \\
\hline diplococos Gram (-) & $6.9 \%$ & $0.0 \% *$ \\
\hline $\begin{array}{l}\text { No. de compañeros } \\
\text { sexuales }\end{array}$ & 2.1 & $1.2^{+}$ \\
\hline
\end{tabular}

*NS

+ Fracción de disparidad (OR) para un compañero: 1.0

OR para dos compañeros: $1.12(\mathrm{p}=0.34)$.

No se encontró diferencia estadísticamente significativa de los eventos previos directamente relacionados con la enfermedad actual entre los grupos de pacientes con EPI y sin EPI en cuanto a: aborto o legrado (21.9\% vs. $30 \%$ ); parto o cesárea (2.7\% vs. $0 \%$ ) y leucorrea $(67.8 \%$ vs. $57.1 \%$ ). El uso actual del DIU (al igual que el uso previo) fue mayor en las pacientes con EPI que en las pacientes $\sin$ EPI (30.1\% vs. $20 \%$, respectivamente).

El síntoma más importante de las pacientes en ambos grupos fue el dolor pélvico. El dolor se presentó en el 100\% de las pacientes del grupo sin EPI y en el $98.5 \%$ del grupo con EPI. La distensión abdominal ocurrió en el $63 \%$ de las pacientes con EPI y en el $50 \%$ de pacientes sin EPI. Solamente refirieron dispareunia un $38.8 \%$ de pacientes del grupo de EPI (Figura 1).
Figura 1

DISTRIBUCION DE LOS SINTOMAS CLINICOS DE ACUERDO CON EI IIA GNOSTICO VISUAL

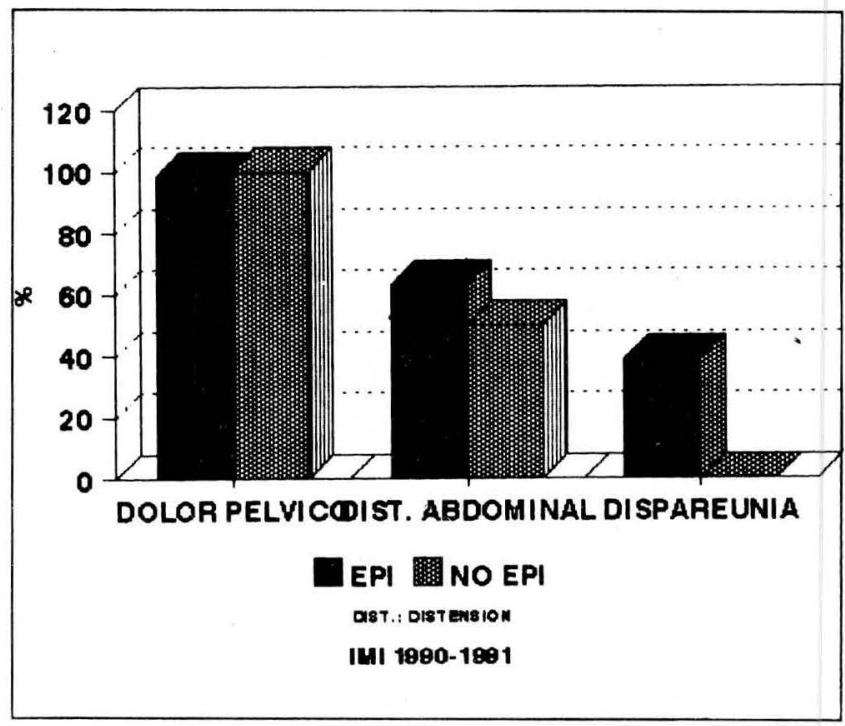

En el examen físico de las pacientes se encontró fiebre en el $37.5 \%$ de pacientes con EPI, mientras que en el segundo grupo se encontró en el $60 \%$ de los casos.

Los signos clínicos considerados como criterios diagnósticos de esta entidad, como son: dolor a la palpación de los anexos, dolor a la movilización del cérvix, dolor en hipogastrio, anexos palpables y flujo endocervical, ocurrieron en una proporción similar de pacientes en ambos grupos (Figura 2).

\section{Figura 2}

DISTRIBUCION DE LOS SIGNOS CLINICOS DE ACUERDO CON EI. DIAGNOSTICO VISUAI

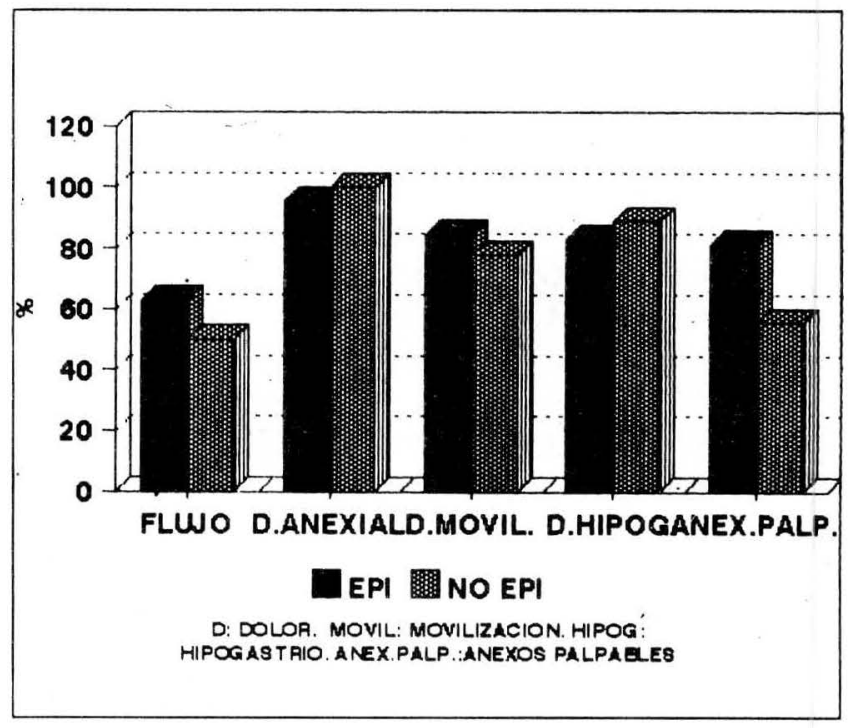

El hemograma se comportó en forma similar en los grupos de pacientes con EPI y sin EPI, mostrando: Leucocitosis en el $60.9 \%$ y $60 \%$, neutrofilia en el $46.8 \%$ y $50 \%$ y $\mathrm{VSG}>15 \mathrm{~mm} / \mathrm{h}$ en el $74.6 \%$ y $70 \%$, respectivamente.

Se realizó ecografía pélvica en el $63.6 \%$ de pacientes con EPI y en el $33.3 \%$ de pacientes del segundo grupo. Se 
encontró masa anexial en el $69 \%$ (29/42) de las pacientes con EPI y en el $66.6 \%(2 / 3)$ de las pacientes sin EPI. La masa fue menor de $8 \mathrm{~cm}$ en el $79.3 \%$ de pacientes con EPI y en el $100 \%$ de pacientes sin EPI.

En la tabla 3 se presentan los diagnósticos clínicos previos a la exploración quirúrgica y se comparan con los hallazgos de la laparoscopia y/o laparotomía en las 76 pacientes. Esta tabla constituye la base para la determinación de la sensibilidad de los hallazgos clínicos para el diagnóstico de la enfermedad pélvica inflamatoria en nuestro estudio.

\section{Tabla 3}

DIAGNOSTICO CLINICO vS. DIAGNOSTICO VISUAL (IMI 1990-1991)

Dx. Visual por laparoscopia o laparotomía*

\begin{tabular}{|lrccc|}
\hline Dx. Clínico & EPI & Otro Dx. Normal & Total \\
\hline EPI & 61 & 8 & 2 & 71 \\
Otro Dx. & 5 & & 5 & \\
Total & 66 & 8 & 2 & 76 \\
\hline
\end{tabular}

* Número de pacientes.

En el 86\% de los casos con sospecha clínica de EPI, ésta fue confirmada. Se encontró un $11 \%$ de falsos positivos con otra patología (apendicitis, ectópico, quiste del ligamento ancho y perforación uterina con pelviperitonitis) y un $2.8 \%$ con pelvis normal. En este grupo de pacientes analizando el diagnóstico clínico de ingreso y el diagnóstico de certeza (establecido por laparoscopia y/o laparotomía), encontramos que los hallazgos clínicos tuvieron una sensibilidad del $92 \%$ y un valor predictivo positivo del $85 \%$ (Tabla 3 ).

En la figura 3 se presenta la distribución de nuestras pacientes de acuerdo con la clasificación laparoscópica de la EPI.

El tratamiento antimicrobiano administrado a este grupo de pacientes se muestra en la tabla 4 .

\section{Tabla 4}

DISTRIBUCION DE LOS ANTIMICROBIANOS EMPLEADOS DE ACUERDO CON EL DIAGNOSTICO VISUAL (IMI 1990-1991)

\begin{tabular}{|lrc|}
\hline Antimicrobianos & EPI (\%) & Sin EPI $(\%)$ \\
\hline Penicilina cristalina + Metronidazol & 67.7 & 80.0 \\
Clindamicina + Gentamicina & 22.6 & 12.5 \\
Cloramfenicol & 23.8 & 11.1 \\
Gentamicina + Nitroimidazol & 1.6 & 12.5 \\
Doxiciclina & 24.6 & 0.0 \\
\hline
\end{tabular}

De las 40 pacientes con EPI que recibieron penicilina cristalina + metronidazol, $15(37.5 \%)$ recibieron además otro esquema antimicrobiano; igual sucedió con 11 de las 14 pacientes que recibieron clindamicina + gentamicina. El cambio de esquema terapéutico no obedeció solamente a falla terapéutica, sino también a inexistencia del medicamento en el Instituto, intolerancia a los medicamentos y otros.
Figura 3

\section{DISTRIBUCION DE PACIENTES DE ACUERDO CON LA CLACIFICACION LAPAROSCOPICA}

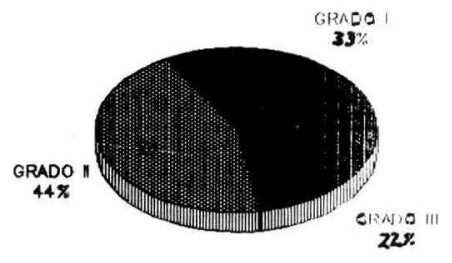

ENFERMEDAD PELVKA INF| AMATOINIA

IMI 1990-1951

Los tratamientos quirúrgicos se muestran en la tabla 5.

Tabla 5

\section{DISTRIBUCION DE LOS TRATAMIENTOS QUIRURGICOS DE ACUERDO CON EL DIAGNOSTICO VISUAL (IMI 1990-1991)}

\begin{tabular}{|lrr|}
\hline Pacientes con EPI & No. & \% \\
\hline Salpingooforectomía & 16 & 24.2 \\
Laparotomía & 15 & 22.7 \\
Lavado peritoneal por laparoscopia & 7 & 10.6 \\
Laparoscopia y laparotomía & 7 & 10.6 \\
Exploración de infundíbulos pélvicos & 2 & 3.0 \\
\hline Pacientes sin EPI & No. & \% \\
\hline Laparoscopia y laparotomía & 5 & 50.0 \\
Salpingooforectomía & 3 & 30.0 \\
Laparotomía & 2 & 20.0 \\
Histerectomía y exploración de & & \\
infundíbulos pélvicos & 1 & 10.0 \\
\hline
\end{tabular}

El tratamiento quirúrgico se encuentra en relación con la severidad de la enfermedad: ninguna paciente con EPI grado I, requirió laparotomía; $28.5 \%$ (10/35) de las pacientes con EPI grados II y III requirieron laparotomía y el $22.8 \%$ de este grupo requirieron salpingooforectomía. Las siete pacientes con EPI que requirieron laparotomía posterior a la laparoscopia tenían enfermedad grados II y III.

Las complicaciones que ocurrieron en las pacientes con EPI, se pueden observar en la tabla 6 :

\section{Discusión}

El promedio de edad de nuestras pacientes con diagnóstico de EPI, fue de 30 años, mientras que en países desarrollados la enfermedad tiende a afectar mujeres cada vez más jóvenes, especialmente, entre los 15 y 25 años (13). En este estudio el promedio de gestaciones de las pacientes fue de 2.6 ; más del $50 \%$ de las pacientes tenían menos de dos hijos 
Tabla 6

COMPLICACIONES DE LAS PACIENTES CON DIAGNOSTICO VISUAL DE EPI (IMI 1990-1991)

\begin{tabular}{|lccc|}
\hline EPI & Absceso tuboovárico & Pelviperitonitis & Peritonitis \\
\hline Grado I & $0 / 18$ & $0 / 18$ & $0 / 18$ \\
Grado II & $6 / 24$ & $0 / 24$ & $1 / 24$ \\
Grado III & $9 / 12$ & $3 / 12$ & $2 / 12$ \\
\hline
\end{tabular}

y no tenían antecedentes de aborto. En otros estudios se ha encontrado que el $74.4 \%$ de pacientes son nulíparas (13).

Varios estudios han revelado que el uso del DIU es más frecuente entre las mujeres con EPI, que en grupos control (13-14). Nosotros también observamos que, tanto el uso actual, como el antecedente de uso del DIU fue mayor en el grupo de pacientes con EPI. Sin embargo, se debe tener en cuenta que además fue el método preferido por todas nuestras pacientes. Respecto a otros métodos de anticoncepción el número de usuarias fue muy escaso, por lo cual no se pueden establecer comparaciones entre los dos grupos (15-16).

Encontramos dos pacientes en el grupo de EPI, con frotis de Gram compatibles con infección gonocóccica, lo cual representa un $13 \%$ de las pacientes a quienes se les realizó este estudio. Esta tasa, aunque similar a la encontrada en otros países (10.8\%) (17), puede representar un subregistro ya que en nuestro medio no se realizan cultivos en forma rutinaria, sin embargo la participación de la $N$. gonorreae en la etiología de la EPI ha sido confirmada por varios autores (18-20).

Encontramos que un $21.9 \%$ de pacientes con EPI, tenían como evento precedente un aborto o un legrado ginecológico y un $2.7 \%$ un parto o una cesárea; sin embargo, en estos datos, no encontramos diferencia significativa con el grupo de pacientes sin EPI. En un estudio de un país en desarrollo se encontró una frecuencia de EPI postaborto del $9.5 \%$ y postparto del $15.3 \%$ (17), mientras que, en países donde el aborto se practica legalmente, la salpingitis aguda se presenta en el $0.5 \%$ de los casos después del procedimiento (13).

Los síntomas clásicos descritos en las mujeres con salpingitis como son el dolor pélvico y la distensión abdominal (8), los encontramos con una alta frecuencia $(98.5 \%$ y $63 \%$, respectivamente), pero se observaron también en el grupo de pacientes con otras patologías, por lo cual son poco específicos para el diagnóstico de EPI. Interesantemente, la dispareunia sólo fue referida por las pacientes con EPI.

Jacobson (8) encontró que los signos clínicos no son de ayuda en el diagnóstico diferencial de esta enfermedad, puesto que las pacientes con otras patologías los presentan con una frecuencia similar. Este autor sólo encontró diferencias significativas en la frecuencia de fiebre y de masas anexiales. En nuestro estudio tampoco observamos diferencias significativas entre los dos grupos con respecto a estos signos. En el grupo de pacientes con EPI, hubo mayor porcentaje de mujeres con anexos palpables al examen físico. Sin embargo, se debe tener precaución en la interpretación de los datos ya que nuestro grupo de pacientes sin EPI es reducido.
Se encontró fiebre solamente en el $37.5 \%$ de pacientes con salpingitis, lo cual coincide con el estudio de Sweet (21), quien informó fiebre en un $40 \%$ de las pacientes. Este autor tampoco encontró diferencias en cuanto a la frecuencia de dolor abdominal, leucorrea, sangrado irregular o síntomas gastrointestinales entre pacientes con diagnóstico visual de EPI y pacientes con pelvis normal.

Los diferentes parámetros del cuadro hemático: leucocitosis, neutrofilia y VSG, no mostraron ninguna diferencia entre pacientes con EPI y pacientes con otras patologías pélvicas, por lo cual son datos inespecíficos. Wolner (22) tampoco observó diferencias en cuanto a la frecuencia de leucocitosis.

Al igual que nuestro estudio, el informe de Jacobson (23), también incluyó todas las pacientes con diagnóstico inicial de EPI y aquellas que ingresaron con un diagnóstico clínico diferente pero la laparoscopia y/o laparotomía revelaron una EPI. Este autor analizó un grupo de 905 pacientes estudiadas a través de un período de 8 años. Jacobson encontró que el diagnóstico clínico de EPI fue confirmado en el $65 \%$ de los casos; fue falso positivo con hallazgo de otras patologías pélvicas en el $12 \%$; falso positivo con pelvis normal en el $23 \%$ y falso negativo (diagnóstico no sospechado clínicamente) en el $15 \%$ de los 623 casos de EPI. El análisis de nuestros datos revela una sensibilidad del diagnóstico clínico del 92\%; un porcentaje similar de falsos positivos con otra patología (11\%) una menor frecuencia de falsos positivos sin otra patología (1.8\%), y de falsos negativos (7.5\%). Estas diferencias pueden deberse tanto, al tamaño de la muestra, como el hecho de que en nuestro Instituto se realizó la laparoscopia a pacientes con mayor número de síntomas o mayor severidad de los mismos que requirieron hospitalización. Jacobson (8) ha encontrado una correlación linear entre el número de variables clínicas encontradas en las pacientes y la confiabilidad del diagnóstico clínico. Sin embargo, debe recordarse que la infección por clamidia (causa frecuente de EPI) puede tener pocos síntomas. En un estudio del Hospital San Ignacio (24) se encontró también un $12 \%$ de diagnósticos clínicos falsos positivos.

El esquema antimicrobiano más utilizado en nuestras pacientes con EPI fue penicilina cristalina + metronidazol, seguido por clindamicina + gentamicina (25). Se utilizó doxiciclina solamente en una cuarta parte de las pacientes.

El enfoque quirúrgico fue conservador: en las pacientes con EPI grado I, no se realizó ninguna laparotomía; de las pacientes con EPI grados II y III sólo requirieron salpingooforectomía unilateral 16 (24.2\% del total de pacientes con EPI) y en las demás pacientes únicamente se realizó lavado de la cavidad peritoneal. Ginsburg publicó 
una serie de pacientes con absceso tuboovárico en quienes se comparó el tratamiento médico con el quirúrgico, refiriendo que en un $80 \%$ de las pacientes tratadas médicamente, el absceso persistía o recurría; en ese estudio las cirugías realizadas fueron: Histerectomía total abdominal en $80 \%$, Salpingooforectomía unilateral en $8 \%$ y drenaje en $12 \%$ (26). Nuestra serie incluye 15 pacientes con absceso tuboovárico confirmado visualmente; ninguna de nuestras pacientes requirió histerectomía.

Sin duda el uso rutinario de la laparoscopia es hasta el momento el procedimiento más confiable para el diagnóstico de salpingitis aguda. Según la experiencia Sueca, se obtiene un diagnóstico correcto en $95 \%$ de los casos con el uso de la laparoscopia. Los estudios que han incluido este procedimiento, demuestran que:
- El diagnóstico clínico de EPI puede ser inexacto.

- La EPI aguda se encuentra frecuentemente en pacientes a quienes se les realiza laparoscopia por otras causas.

- La laparoscopia es una forma segura de hacer un diagnóstico visual.

- La laparoscopia es un método excelente para obtener cultivos directos.

Sin embargo, la laparoscopia es un método costoso y poco práctico dado el número tan alto de pacientes con EPI. Por estas razones las bases diagnósticas siguen siendo los criterios clínicos, pero se están estudiando procedimientos más sencillos y/o económicos para establecer el diagnóstico de la enfermedad.

\section{BIBLIOGRAFIA}

1. Pastorek II JG. Pelvic inflammatory disease and tubo-ovarian abscess. Obstet. Gynecol. Clin. North. Am. 1989; 16. 347-361.

2. Fonnegra A., Guzmán RD. Cincuenta años (50) de enfermedad pélvica inflamatoria en el Hospital San Juan de Dios - Bogotá. Rev. Col. Obstet. Ginecol. 1966; 17: 155-163.

3. Espinoza y Espinoza A., Handszer B., Alba L. Enfermedad inflamatoria pélvica. Comentarios a dos años y medio de estadística en el Servicio de Ginecología. Hospital San Juan de Dios - Bogotá, D.E. Rev. Col. Obstet. Ginecol. 1961; 12: 533-555.

4. Fonnegra A., Rocha-Posada H., Handszer B. Enfermedad pélvica inflamatoria en ginecología. Rev. Col. Obstet. Ginecol. 1967; 18: 431-439.

5. Ledger WJ. The next 10 years in the treatment of pelvic infections. Am. J. Med. 1985; 78(Suppl. 6B): 194-202.

6. Mead P., Louria D. Antibióticos en infecciones pelvianas. Clin. Obstet. Ginecol. 1969; 219-237.

7. Lozano A., Peralta MT. et al. Morbimortalidad del aborto séptico en el IMI. Rev. Col. Obstet. Ginecol. 1981; 32: 104-134.

8. Jacobson L., Weström L. Objectivized diagnosis of acute pelvic inflammatory disease. Am. J. Obstet. Gynecol. 1969; 105: 10881098.

9. Sweet RL., Draper DL., Schachter J. et al. Microbiology and pathogenesis of acute salpingitis as determined by laparoscopy: what is the appropriate site to sample? Am. J. Obstet. Gynecol. 1980; 138: 985-989.

10. Chow AW., Malkasian KL., Marshall JR. et al. The bacteriology of acute pelvic inflammatory disease. Value of cul-de-sac cultures and relative importance of gonococci and other aerobic or anaerobic bacteria. Am. J. Obstet. Gynecol. 1975; 122: 876-879.

11. Sweet RL. Pelvic inflammatory disease and infertility in women. Infect. Dis. Clin. North. Am. 1987; 1: 199-215.

12. Hager WD., Eschembach DA., Spence MR. et al. Editorial: Criteria for diagnosis and grading of salpingitis. Obstet. Gynecol. 1983; 61: $113-114$
13. Weström L. Incidence, prevalence, and trend of acute pelvic inflammatory disease and its consequences in industrialized countries. Am. J. Obstet. Gynecol. 1980; 138: 880-890.13.

14. Burkman RT. Intrauterine device use and the risk of pelvic inflammatory disease. Am. J. Obstet. Gynecol. 1980; 138: 861-863.

15. Kelaghan J., Rubin GL., Ory HW. et al. Barrier-method contraceptives and pelvic inflammatory disease. JAMA 1982; 248: 184-187.

16. Svensson L., Weström L., Mardh P-A. Contraceptives and acute salpingitis. JAMA 1984; 251: 2553-2555.

17. Muir BA., Belsey MA. Pelvic inflammatory disease and its consequences in the developing world. Am. J. Obstet. Gynecol. 1980; 138: 913-927.

18. Ratnam AV.,Din SN., Chatterjee TK. Gonococcal infection in women with pelvic inflammatory disease in Lusaka, Zambia. Am. J. Obstet. Gynecol. 1980; 138: 965-967.

19. Eschenbach DA. Epidemiology and diagnosis of acute pelvic inflammatory disease. Obstet. Gynecol. 1980; 55(Suppl): 142S.

20. Eschembach DA., Holmes KK. Acute pelvic inflammatory disease: currents concepts of pathogenesis, etiology, and management. Clin. Obstet. Gynecol. 1975; 18: 35 .

21. Sweet R., Gibbs R. Infectious Diseases of the Female Genital Tract. Williams \& Wilkins, Baltimore 1990.

22. Wolner, Hansen et al. Laparoscopy in women with chlamydial infection and pelvic pain: a comparison of patients with and without salpingitis. Obstet. Gynecol. 1983; 61: 299-303.

23. Jacobson L: Differential diagnosis of acute pelvic inflammatory disease. Am. J. Obstet. Gynecol. 1980; 138: 1006-1011.

24. Angulo A., Moreno B., Mora E. Tratamiento de enfermedad pélvica inflamatoria con Metronidazol y/o Penicilina. Rev. Col. Obstet. Ginecol. 1984; 36: 123-131.

25. Walters MD., Gibbs RS. A randomized comparison of gentamicinclindamicin in the treatment of pelvic inflammatory disease. Obstet. Gynecol. 1990; 75: 867-872.

26. Ginsburg D., Stern J., Hamod K. et al. Tuboovarian abscess. A retrospective review. Am. J. Obstet. Gynecol. 1980; 138: 1055. 